\title{
Effect of Feedback Delay on Downlink Amplify-and-Forward Relaying with Beamforming
}

\author{
Himal A. Suraweera*, Theodoros A. Tsiftsis ${ }^{\dagger}$, George K. Karagiannidis ${ }^{\dagger}$ and Michael Faulkner ${ }^{\ddagger}$ \\ *Department of Electrical \& Computer Engineering, National University of Singapore, Singapore 119260 \\ $\dagger$ Department of Electrical \& Computer Engineering, Aristotle University of Thessaloniki, 54124 Thessaloniki, Greece \\ $\ddagger$ Center for Telecommunications and Microelectronics, Victoria University, Melbourne, Victoria 8001, Australia \\ E-mail: elesaha@nus.edu.sg, \{thtsif,geokarag\}@auth.gr, mike.faulkner@vu.edu.au
}

\begin{abstract}
In relay networks, partial channel state information at the transmitter due to feedback delay can severely degrade the beamforming performance. In this paper, the decremental effect of transmit beamforming with feedback delay on the downlink performance of a two hop Amplify-and-Forward (AF) relay network over Rayleigh fading channels is investigated. The source is equipped with multiple antennas while the relay and the destination has a single antenna. We have derived closed-form expressions for the outage probability, probability density function of the received signal-to-noise ratio (SNR) at the destination and the average bit error rate (BER). To gain further insights, outage probability and average BER approximations at high SNR are also presented. Numerical results supported by simulations are provided to illustrate the decremental effects feedback delay on the performance of the considered AF relay system.
\end{abstract}

\section{INTRODUCTION}

Relay deployment to assist source-destination communications has been shown to offer significant performance benefits [1], including cellular coverage enhancement. The two most common relaying protocols are Decode-and-Forward (DF) and Amplify-and-Forward (AF). AF may use the knowledge of the source to relay channel to assist signal amplification without decoding [2], [3]. These kind of relays are widely identified as channel state information (CSI) assisted relays.

Transmit beamforming has been shown to significantly improve the performance of point-to-point multi-antenna systems. When perfect CSI is available at the transmitter (CSIT), beamforming schemes maximizes the received signal-to-noise ratio (SNR). Such knowledge is provided by feedback of CSI from the receiver to the transmitter. Practical limitations such as channel estimation errors, limited feedback and feedback delay can give rise to partial CSIT. These limitations degrade the beamforming performance [4]. The performance of pointto-point beamforming systems under partial CSIT has been widely investigated in the literature. See for example [4], [5] and references therein.

Recent works such as [6] has demonstrated significant benefits of deploying multiple antennas in relaying systems. A practical transmission scheme for $\mathrm{AF}$ relaying systems employing multiple antennas is transmit beamforming. In

This research is supported under the Australian Research Council's Discovery funding scheme (DP0774689). a two hop AF relay system, one method of beamforming considers the cascaded source-to-relay and relay-to-destination links [7], [8]. The beamforming vector is chosen considering noise amplification at the relay. Another method is to perform hop-by-hop beamforming [9]-[13] where source-relay and relay-destination links are considered separately to decide on the beamforming vector. The performance of beamforming against relay selection considering both unlimited and limited feedback has been studied in [7]. In [8], distributed beamforming and a power allocation scheme for AF relaying have been proposed. In [9], the performance of the DF protocol in multiple relay channels with maximal ratio combining (MRC) and transmit beamforming has been analyzed. In [10], the performance of a two hop relay network with transmit beamforming at the source and MRC at the destination has been analyzed. In [11], a pilot emitting scheme is proposed for hop-by-hop beamforming in AF relaying and the channel estimation performance has been investigated. In [12], the outage performance with beamforming considering only limited feedback has been studied. However, so far to the authors' best knowledge, the effect of delayed CSI feedback on the beamforming performance in AF relay systems has not been investigated.

Most of the above research consider cases where a number of antennas are placed both at the transmitter and at the receiver (i.e., MIMO beamforming or transmit-receive diversity) or communication between the two ends is achieved using a number of relays (i.e., distributed transmit beamforming). Very recently, in [13], a practical scenario in which a multiantenna equipped source is communicating to a destination via a relay is considered. Both the destination and relay are equipped with a single antenna respectively. This scenario can be directly applied to current cellular networks where the use of multiple antennas in a base station is reasonable, but the use of multiple antennas at mobile terminals and or relays may be prohibitive due to the terminal size and power constraints. Although the above described case is of high practical interest, the authors in [13] have limited their study to an ideal situation, where the source has perfect channel knowledge. Furthermore, outage probability expressions when only specific number of antennas are employed at the source are presented. In wireless system studying the error performance is also important. In 


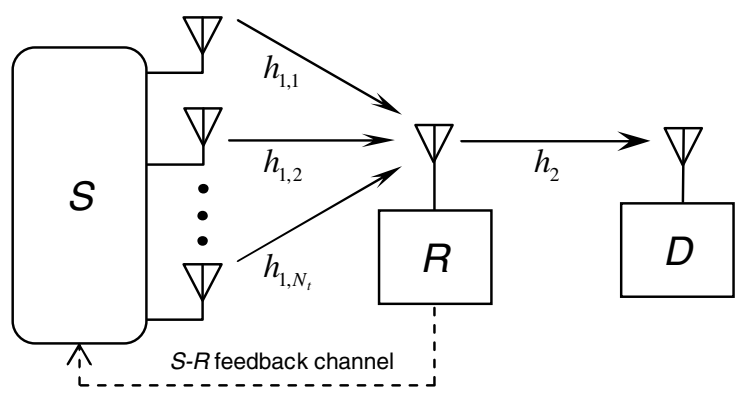

Fig. 1. Wireless communication system with AF relay beamforming.

relay systems, delays in conveying the transmit beamforming information vector from the relay to the source can degrade the performance of the transmit beamforming.

In this paper, we consider a two hop AF relay network where multiple antennas at the source are used for beamforming. We investigate the impact of partial CSIT due to delayed feedback on the end-to-end (e2e) system performance and derive closed-form expressions for the system's outage probability and the average bit error rate (BER) applicable for a range of modulation schemes. To gain valuable insights, in the high SNR regime, we also present asymptotic outage probability and average BER expressions.

This paper is organized as follows. Section II describes the system model. The system performance analysis is carried out in Section III providing new expressions for the cdf/pdf of the e2e SNR and an exact/asymptotic BER study. Numerical results supported by simulations are presented in Section IV to verify the new results. Finally, we conclude in Section V.

\section{SySTEM MODEL}

Consider a wireless communications system where a source, $S$, equipped with $N_{t}$ antennas communicates with a destination, $D$, through a relay, $R$ as shown in Fig. 1. Both the relay and the destination is equipped with a single antenna. This model can represent the downlink of a mobile communication network in which a mobile station is used to act as a relay for communications between a multi-antenna equipped base station and another mobile station. It is assumed that the source does not have a direct link to the destination, which may result from high shadowing between the source and the destination.

The communication from $S$ to $D$ via the relay $R$ takes place in two time slots. In the first time slot, $S$ beamforms its signal to $R$. The received signal at the relay can be written as

$$
y_{R}(t)=\mathbf{w}^{H}\left(t \mid T_{d}\right)(t) \mathbf{h}_{1}(t) x(t)+n_{1}(t)
$$

where $x(t)$ is the data symbol, $\mathbf{h}_{1}(t)=\left[h_{1,1}(t), \ldots, h_{1, N_{t}}\right]^{T}$ is the channel vector from $S$ to $R$ with Rayleigh fading entries, $n_{1}(t)$ is the white Gaussian noise with one sided power spectrum density $\sigma_{1}^{2}$ at the relay and $(\cdot)^{T},(\cdot)^{H}$ denote the transpose and Hermitian transpose, respectively. The beamforming vector, $\mathbf{w}(t)$ satisfies $\mathbf{w}^{H}(t) \mathbf{w}(t)=1$ and is calculated from the outdated channel $\mathbf{h}_{1}\left(t-T_{d}\right), T_{d}$ being the feedback delay. We assume that the relay estimates the received equivalent channel, $\mathbf{w}^{H}\left(t \mid T_{d}\right) \mathbf{h}_{1}(t)$ perfectly. The received signal $y_{R}(t)$ is multiplied by a gain, $G$, and retransmitted to the destination in the second time slot. The received signal at the destination is given by

$$
\begin{aligned}
y_{D}(t) & =h_{2}(t) G y_{R}(t)+n_{2}(t) \\
& =h_{2}(t) G\left(\mathbf{w}^{H}\left(t \mid T_{d}\right) \mathbf{h}_{1}(t) x+n_{1}(t)\right)+n_{2}(t)
\end{aligned}
$$

where $P_{2}$ is the relay transmit power, $h_{2}$ is the complex Rayleigh channel from the relay to the destination and $n_{2}(t)$ is the AWGN with one sided power spectral density $\sigma_{2}^{2}$. For CSI assisted AF, the gain $G$ is given by [2]

$$
G^{2}=\frac{1}{P_{1}\left|\mathbf{w}^{H}\left(t \mid T_{d}\right) \mathbf{h}_{1}\right|^{2}+\sigma_{1}^{2}}
$$

The resulting e2e SNR at the destination can be written as

$$
\gamma_{e q}=\frac{\frac{P_{1}\left|\mathbf{w}^{H}\left(t \mid T_{d}\right) \mathbf{h}_{1}(t)\right|^{2}}{\sigma_{1}^{2}} \frac{P_{2}\left|h_{2}\right|^{2}}{\sigma_{2}^{2}}}{\frac{P_{2}\left|h_{2}\right|^{2}}{\sigma_{2}^{2}}+\frac{1}{G^{2} \sigma_{1}^{2}}} .
$$

and can be simplified as

$$
\gamma_{e q 1}=\frac{\gamma_{1} \gamma_{2}}{\gamma_{1}+\gamma_{2}+1}
$$

where $\gamma_{1}=\left|\mathbf{w}^{H}\left(t \mid T_{d}\right) \mathbf{h}_{1}(t)\right|^{2} \bar{\gamma}_{1}, \gamma_{2}=\left|h_{2}\right|^{2} \bar{\gamma}_{2}$, and $\bar{\gamma}_{i}=\frac{P_{i}}{\sigma_{i}^{2}}$ for $i=1,2$. However, the form of the e2e SNR in (5) is not easily tractable for further analysis [2]. Fortunately, (5) can be tightly upper bounded by

$$
\gamma_{e q 1}<\gamma_{e q 2}=\frac{\gamma_{1} \gamma_{2}}{\gamma_{1}+\gamma_{2}}
$$

The form of $\gamma_{e q 2}$ has the advantage of mathematical tractability over that in (5) and it is a tight upper bound for $\gamma_{e q 1}$, specially at medium to high average SNR.

\section{Performance Analysis}

In this section, we derive important performance measures for the two hop system under investigation. This includes the outage probability, asymptotic outage probability and the error performance.

For example, in order to study the outage probability of (5) and (6), it is necessary to obtain the distribution of the e2e SNR $\gamma_{\text {eqi }}, i=1,2$ of a general form

$$
Y=\frac{\gamma_{1} \gamma_{2}}{\gamma_{1}+\gamma_{2}+c}
$$

where $c \geq 0$ is a constant.

\section{A. Outage Probability and the e2e SNR pdf}

The outage probability, $P_{o}$ is an important quality of service (QoS) measure defined as the probability that $\gamma_{e q 1}$ drops below an acceptable SNR threshold $\gamma_{\text {th }}$, or

$$
P_{o}=\operatorname{Pr}\left(\gamma_{e q 1}<\gamma_{\mathrm{th}}\right)=F_{\gamma_{e q 1}}\left(\gamma_{\mathrm{th}}\right) \text {. }
$$

To derive the outage probability of $\gamma_{e q 1}$, conditioning on $\gamma_{1}$, we first express the cumulative distribution function (cdf) of $Y$ as

$$
F_{Y}\left(\gamma_{\text {th }}\right)=\int_{0}^{\infty} \operatorname{Pr}\left(\frac{z \gamma_{2}}{z+\gamma_{2}+c}<\gamma_{\text {th }}\right) p_{\gamma_{1}}(z) d z
$$


After applying some algebraic manipulations to (9), $F_{Y}\left(\gamma_{\mathrm{th}}\right)$ can be re-expressed as

$$
\begin{aligned}
& F_{Y}\left(\gamma_{\mathrm{th}}\right)= \\
& \quad 1-\int_{0}^{\infty} p_{\gamma_{1}}\left(x+\gamma_{\mathrm{th}}\right)\left(1-F_{\gamma_{2}}\left(\gamma_{\mathrm{th}}+\frac{\gamma_{\mathrm{th}}^{2}+c \gamma_{\mathrm{th}}}{x}\right)\right) d x
\end{aligned}
$$

where $p_{\gamma_{1}}(\cdot)$ and $F_{\gamma_{2}}(\cdot)$ denote the probability density function (pdf) of $\gamma_{1}$ and the cdf of $\gamma_{2}$ respectively.

To obtain $F_{Y}(y)$, we need expressions for the pdf of $\gamma_{1}$ and the cdf of $\gamma_{2}$. When the following widely adopted channel feedback delay model is employed [5]

$$
\mathbf{h}_{1}(t)=\rho_{d} \mathbf{h}_{1}\left(t-T_{d}\right)+\mathbf{e}(t)
$$

where $\rho_{d}=J_{0}\left(2 \pi f_{d} T_{d}\right)$ is the normalized correlation coefficient specified from the Jake's model between $\mathbf{h}_{1 j}(t)$ and $\mathbf{h}_{1 j}\left(t-T_{d}\right)$ with $j=1, \ldots, N_{t}, f_{d}$ is the Doppler frequency, $J_{0}(\cdot)$ is the zeroth-order Bessel function of the first kind [15, Eq. (9.1.18)] and $\mathbf{e}(t)$ is an error vector.

The pdf of $\gamma_{1}$ using [5, Eq. (15)], i.e. the case of full-rate feedback, can be written as

$$
\begin{aligned}
p_{\gamma_{1}}(x) & =\frac{1}{\bar{\gamma}_{1}^{N_{t}}} \sum_{i=0}^{N_{t}-1} \frac{\left(\begin{array}{c}
N_{t}-1 \\
i
\end{array}\right)}{\left(N_{t}-i-1\right) !}\left(\left|\rho_{d}\right|^{2}\right)^{N_{t}-i-1} \\
& \times\left(\bar{\gamma}_{1}\left(1-\left|\rho_{d}\right|^{2}\right)\right)^{i} x^{N_{t}-i-1} e^{-\frac{x}{\bar{\gamma}_{1}}}
\end{aligned}
$$

Since we assume a Rayleigh distribution for the $R-D$ link, the cdf of $\gamma_{2}$ is simply given by

$$
F_{\gamma_{2}}(x)=1-e^{-\frac{x}{\bar{\gamma}_{2}}},
$$

Substituting (12) and (13) into (10) we obtain

$$
\begin{aligned}
F_{Y}\left(\gamma_{\mathrm{th}}\right) & =1-\frac{e^{-\left(\frac{1}{\bar{\gamma}_{1}}+\frac{1}{\bar{\gamma}_{2}}\right) \gamma_{\mathrm{th}}}}{\bar{\gamma}_{1}^{N_{t}}} \sum_{i=0}^{N_{t}-1} \frac{\left(\begin{array}{c}
N_{t}-1 \\
i
\end{array}\right)}{\left(N_{t}-i-1\right) !} \\
& \times\left(\left|\rho_{d}\right|^{2}\right)^{N_{t}-i-1}\left(\bar{\gamma}_{1}\left(1-\left|\rho_{d}\right|^{2}\right)\right)^{i} \\
& \times \int_{0}^{\infty}\left(x+\gamma_{\mathrm{th}}\right)^{N_{t}-i-1} e^{-\frac{x}{\bar{\gamma}_{1}}-\frac{\gamma_{\mathrm{th}}^{2}+c \gamma_{\mathrm{th}}}{x \bar{\gamma}_{2}}} d x
\end{aligned}
$$

Now expressing $\left(x+\gamma_{\mathrm{th}}\right)^{N_{t}-n-1}$ using the binomial theorem and next with the help of the identity [14, Eq. (3.471.9)], the integral in (14) can be solved in closed-form. Therefore, $F_{Y}\left(\gamma_{\text {th }}\right)$ can be expressed as

$$
\begin{aligned}
F_{Y}\left(\gamma_{\mathrm{th}}\right) & =1-\frac{2 e^{-\left(\frac{1}{\bar{\gamma}_{1}}+\frac{1}{\bar{\gamma}_{2}}\right) \gamma_{\mathrm{th}}}}{\bar{\gamma}_{1}^{N_{t}}}\left(\left|\rho_{d}\right|^{2} \gamma_{\mathrm{th}}\right)^{N_{t}-1} \\
& \times \sum_{i=0}^{N_{t}-1} \frac{\left(\begin{array}{c}
N_{t}-1 \\
i
\end{array}\right)\left(\frac{\bar{\gamma}_{1}\left(1-\left|\rho_{d}\right|^{2}\right)}{|\rho|^{2} \gamma_{\mathrm{th}}}\right)^{i}}{\left(N_{t}-i-1\right) !} \sum_{j=0}^{N_{t}-i-1} \frac{\left(\begin{array}{c}
N_{t}-i-1 \\
j
\end{array}\right)}{\gamma_{\mathrm{th}}^{j}} \\
& \times\left(\frac{\bar{\gamma}_{1}\left(\gamma_{\mathrm{th}}^{2}+c \gamma_{\mathrm{th}}\right)}{\bar{\gamma}_{2}}\right)^{\frac{j+1}{2}} \mathcal{K}_{j+1}\left(2 \sqrt{\frac{\gamma_{\mathrm{th}}^{2}+c \gamma_{\mathrm{th}}}{\bar{\gamma}_{1} \bar{\gamma}_{2}}}\right)
\end{aligned}
$$

where $\mathcal{K}_{\nu}(\cdot)$ is the $\nu$ th-order modified Bessel function of the second kind. Note that when there is no feedback delay $\left(T_{d}=\right.$
$0)$, the cdf of $Y$ can be obtained from (15) by substituting $i=0$ and $\left|\rho_{d}\right|^{2}=1$. This is given by

$$
\begin{aligned}
& F_{Y}\left(\gamma_{\text {th }}\right)=1-\frac{2 e^{-\left(\frac{1}{\bar{\gamma}_{1}}+\frac{1}{\bar{\gamma}_{2}}\right) \gamma_{\text {th }}}}{\left(N_{t}-1\right) ! \bar{\gamma}_{1}^{N_{t}}} \sum_{j=0}^{N_{t}-1}\left(\begin{array}{c}
N_{t}-1 \\
j
\end{array}\right) \\
& \times \gamma_{\text {th }}^{N_{t}-j-1}\left(\frac{\bar{\gamma}_{1}\left(\gamma_{\text {th }}^{2}+c \gamma_{\text {th }}\right)}{\bar{\gamma}_{2}}\right)^{\frac{j+1}{2}} \mathcal{K}_{j+1}\left(2 \sqrt{\frac{\gamma_{\text {th }}^{2}+c \gamma_{\text {th }}}{\bar{\gamma}_{1} \bar{\gamma}_{2}}}\right)
\end{aligned}
$$

The pdf of $Y$ can now be obtained by differentiating $F_{Y}\left(\gamma_{\text {th }}\right)$ with respect to $\gamma_{\text {th }}$. Therefore, the pdf of the received e2e SNR is given by

$$
\begin{aligned}
& f_{Y}(y)=\frac{2}{\bar{\gamma}_{1}^{N_{t}}}\left(\left|\rho_{d}\right|\right)^{N_{t}-1} \sum_{i=0}^{N_{t}-1} \sum_{j=0}^{N_{t}-i-1} \frac{\left(\begin{array}{c}
N_{t}-1 \\
i
\end{array}\right)\left(\begin{array}{c}
N_{t}-i-1 \\
j
\end{array}\right)}{\left(N_{t}-i-1\right) !}(17) \\
& \times\left(\frac{\bar{\gamma}_{1}}{\bar{\gamma}_{2}}\right)^{\frac{j+1}{2}}\left(\frac{\bar{\gamma}_{1}\left(1-\left|\rho_{d}\right|^{2}\right)}{\left|\rho_{d}\right|^{2}}\right)\left\{e^{-\left(\frac{1}{\bar{\gamma}_{1}}+\frac{1}{\bar{\gamma}_{2}}\right) \gamma_{\mathrm{th}}}\right. \\
& \times \gamma_{\mathrm{th}}^{N_{t}-j-i-1}\left(c \gamma_{\mathrm{th}}+\gamma_{\mathrm{th}}^{2}\right)^{\frac{1+j}{2}} \mathcal{K}_{j+1}\left(2 \sqrt{\frac{\gamma_{\mathrm{th}}^{2}+c \gamma_{\mathrm{th}}}{\bar{\gamma}_{1} \bar{\gamma}_{2}}}\right) \\
& \left.\times\left[\left(\frac{1}{\bar{\gamma}_{1}}+\frac{1}{\bar{\gamma}_{2}}\right)-\frac{(1+j)\left(c+2 \gamma_{\mathrm{th}}\right)}{2\left(c \gamma_{\mathrm{th}}+\gamma_{\mathrm{th}}^{2}\right)}+\frac{1+i+j-\gamma_{\mathrm{th}}}{2}\right]\right\} \\
& +\frac{e^{-\left(\frac{1}{\bar{\gamma}_{1}}+\frac{1}{\bar{\gamma}_{2}}\right) \gamma_{\mathrm{th}} \gamma_{\mathrm{th}}^{N_{t}-i-j-1}\left(c+2 \gamma_{\mathrm{th}}\right)\left(c \gamma_{\mathrm{th}}+\gamma_{\mathrm{th}}^{2}\right)^{\frac{1+j}{2}}}}{\bar{\gamma}_{1} \bar{\gamma}_{2} \sqrt{\frac{\gamma_{\mathrm{th}}^{2}+c \gamma_{\mathrm{th}}}{\bar{\gamma}_{1} \bar{\gamma}_{2}}}} \\
& \left(\mathcal{K}_{j}\left(2 \sqrt{\frac{\gamma_{\mathrm{th}}^{2}+c \gamma_{\mathrm{th}}}{\bar{\gamma}_{1} \bar{\gamma}_{2}}}\right)+\mathcal{K}_{j+2}\left(2 \sqrt{\frac{\gamma_{\mathrm{th}}^{2}+c \gamma_{\mathrm{th}}}{\bar{\gamma}_{1} \bar{\gamma}_{2}}}\right)\right)
\end{aligned}
$$

\section{B. Outage Probability at high SNR}

Although (15) is exact and valid for any SNR, it is difficult to obtain insights such as the effect of parameters $N_{t}$ and $\rho_{d}$ on the outage probability. Therefore, we now analyze the system's e2e asymptotic outage probability. The outage probability at high SNR is given by

$$
P_{o}^{\infty} \simeq\left(\frac{1+\kappa}{\kappa}-\left(1-\left(1-\left|\rho_{d}\right|^{2}\right)^{N_{t}-1}\right)\right) \frac{\gamma_{\mathrm{th}}}{\bar{\gamma}_{1}}
$$

where $\kappa=\frac{\bar{\gamma}_{2}}{\bar{\gamma}_{1}}$.

Proof: See Appendix.

\section{Average BER}

We now analyze the average BER performance of the AF system introduced in Section II. We consider $\gamma_{e q 2}$ instead of (5) for mathematical tractability purposes and because it provides a tight upper bound for CSI assisted AF relaying in the medium to high SNR regime. For many general modulation formats (see below), the average BER can be expressed as

$$
P_{b}=a \mathbb{E}_{\gamma}[Q(\sqrt{b \gamma})],
$$

where $\mathbb{E}[\cdot]$ denotes the statistical expectation operator, $a, b>$ 0 , and $Q(x)=\frac{1}{\sqrt{2 \pi}} \int_{x}^{\infty} e^{-\frac{y^{2}}{2}} d y$ is the Gaussian $Q$-function. For binary PSK $(a, b)=(1,2)$, binary frequency shift keying with orthogonal signaling $(a, b)=(1,1)$ gives the exact BER 


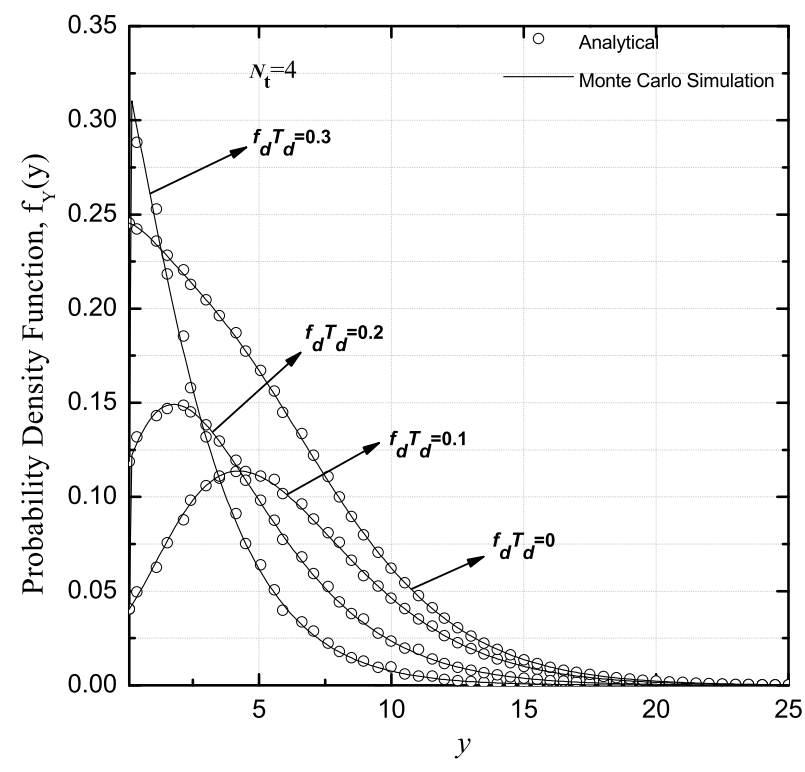

Fig. 2. Pdf of the e2e SNR versus feedback delay $f_{d} T_{d}$ for $N t=4, \bar{\gamma}_{1}=5$ $\mathrm{dB}, \bar{\gamma}_{2}=15 \mathrm{~dB}$, and $c=1$.

and for $M$-PSK $(a, b)=\frac{1}{\log _{2} M}, \log _{2} M \sin ^{2} \frac{\pi}{M}$ can be used to approximate the BER.

Using integration by parts it can be shown that

$$
P_{b}=\frac{a}{\sqrt{2 \pi}} \int_{0}^{\infty} F_{\gamma_{e q 2}}\left(\frac{t^{2}}{b}\right) e^{-\frac{t^{2}}{2}} d t .
$$

Substituting (15) into (20), $P_{b}$ can be expressed as

$$
\begin{aligned}
P_{b} & =\frac{a}{2}-\frac{\sqrt{2} a\left(\left|\rho_{d}\right|^{2}\right)^{N_{t}-1}}{\sqrt{\pi}\left(b \bar{\gamma}_{1}\right)^{N_{t}}} \sum_{i=0}^{N_{t}-1} \frac{\left(\begin{array}{c}
N_{t}-1 \\
i
\end{array}\right)}{\left(N_{t}-i-1\right) !} \\
& \times\left(\frac{b \bar{\gamma}_{1}\left(1-\left|\rho_{d}\right|^{2}\right)}{|\rho|^{2}}\right)^{i N_{t}-i-1} \sum_{j=0}^{\infty}\left(\begin{array}{c}
N_{t}-i-1 \\
j
\end{array}\right)\left(\frac{\bar{\gamma}_{1}}{\bar{\gamma}_{2}}\right)^{\frac{j+1}{2}} \\
& \times \int_{0}^{\infty} t^{2\left(N_{t}-i\right)} e^{-\left(\frac{1}{b \bar{\gamma}_{1}}+\frac{1}{b \bar{\gamma}_{2}}+\frac{1}{2}\right) t^{2}} \mathcal{K}_{j+1}\left(\frac{2 t^{2}}{b \sqrt{\bar{\gamma}_{1} \bar{\gamma}_{2}}}\right) d t
\end{aligned}
$$

Applying a simple variable transformation and using [14, Eq. (6.621.3)], average BER has a closed-form solution given by (22) at the top of next page.

When there is no feedback delay, the average BER simplifies to

$$
\begin{aligned}
P_{b} & =\frac{a}{2}-\frac{2 a \sqrt{2 b}}{\left(N_{t}-1\right) ! N_{t} ! \bar{\gamma}_{1}^{N_{t}} \bar{\gamma}_{2}} \sum_{j=0}^{N_{t}-1}\left(\begin{array}{c}
N_{t}-1 \\
j
\end{array}\right) \\
& \times\left(\frac{4}{\bar{\gamma}_{2}}\right)^{j} \Gamma\left(N_{t}+j+\frac{3}{2}\right) \Gamma\left(N_{t}-j-\frac{1}{2}\right) \\
& \times \frac{{ }_{2} F_{1}\left(N_{t}+j+\frac{3}{2}, j+\frac{3}{2} ; N_{t}+1 ; \frac{\frac{1}{\bar{\gamma}_{1}}+\frac{1}{\bar{\gamma}_{2}}+\frac{b}{2}-\frac{2}{\sqrt{\bar{\gamma}_{1} \gamma_{2}}}}{\bar{\gamma}_{1}+\frac{1}{\bar{\gamma}_{2}}+\frac{b}{2}+\frac{2}{\sqrt{\gamma_{1} \bar{\gamma}_{2}}}}\right)}{\left(\frac{1}{\bar{\gamma}_{1}}+\frac{1}{\bar{\gamma}_{2}}+\frac{b}{2}+\frac{2}{\sqrt{\bar{\gamma}_{1} \bar{\gamma}_{2}}}\right)^{N_{t}+j+\frac{3}{2}}} .
\end{aligned}
$$

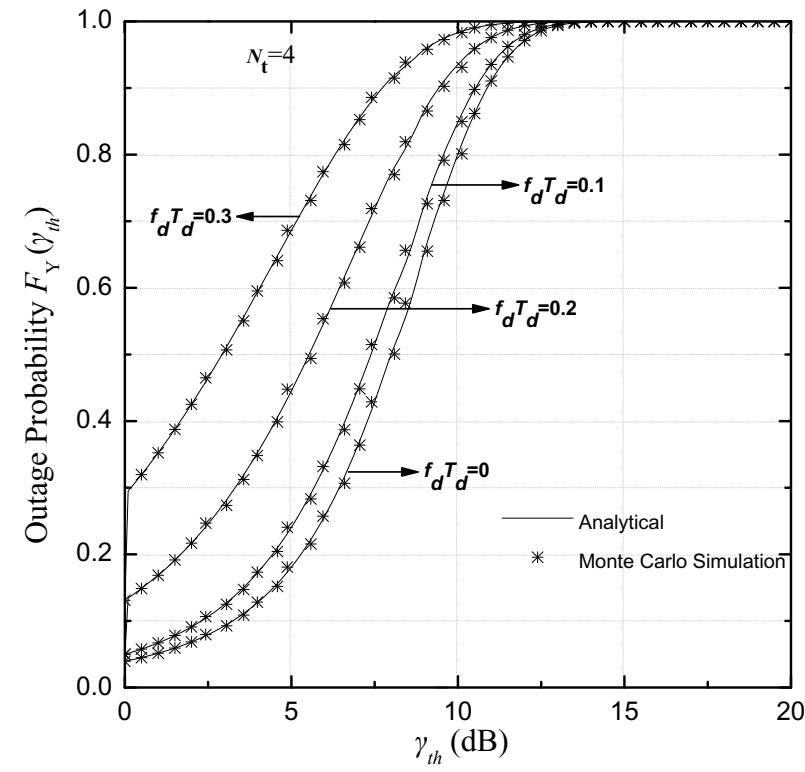

Fig. 3. Outage probability for $N_{t}=4, \bar{\gamma}_{1}=5 \mathrm{~dB}, \bar{\gamma}_{2}=15 \mathrm{~dB}$, and $c=1$.

\section{Average BER at high SNR}

Asymptotic analysis on relaying systems are important since such studies provide valuable insights that are useful to design engineers. At sufficiently high SNRs, diversity gain, $G_{d}$, defines the slope of the average BER against average SNR in a log-log scale while array gain, $G_{a}$, determines the shift of the curve with respect to the average BER curve of $\bar{\gamma}_{1}^{-G_{d}}$.

It is shown in [16] that at high SNRs, the error performance can be calculated based on the behavior of the pdf of the instantaneous channel power gain. We write $f(x)=O[g(x)]$, $x \rightarrow x_{0}$, if $\lim _{x \rightarrow x_{0}} \frac{f(x)}{g(x)}=0$. Following [16, Prop. 1] the average BER at high SNRs can be closely approximated as

$$
P_{b}^{\infty}=\frac{2^{q} a \delta \Gamma\left(q+\frac{3}{2}\right)}{\sqrt{\pi}(q+1)}\left(b \bar{\gamma}_{1}\right)^{-(q+1)}+O\left(\bar{\gamma}_{1}^{-(q+1)}\right),
$$

where $q=0$ and

$$
\delta=\left(\frac{1+\kappa}{\kappa}-\left(1-\left(1-\left|\rho_{d}\right|^{2}\right)^{N_{t}-1}\right)\right)
$$

Eq. (24) implies that $G_{a}$ and $G_{d}$ of the system can be written as

$$
G_{a}=b\left(\frac{2^{q} a \delta \Gamma\left(q+\frac{3}{2}\right)}{\sqrt{\pi}(q+1)}\right)^{-\frac{1}{q+1}}
$$

and

$$
G_{d}=1
$$

\section{NumERICAL AND Simulation RESUlts}

In this section, we analyze the beamforming two hop AF relay system due to feedback delay, and confirm the derived analytical results through comparison with Monte Carlo simulations.

Fig. 2 presents the analytical and simulated pdf for various feedback delays $f_{d} T_{d}=0,0.1,0.2$ or 0.3 or equivalently the 


$$
\begin{aligned}
P_{b} & =\frac{a}{2}-\frac{2 a \sqrt{2 b}\left(\left|\rho_{d}\right|^{2}\right)^{N_{t}-1}}{\bar{\gamma}_{1}^{N_{t}} \bar{\gamma}_{2}} \sum_{i=0}^{N_{t}-1} \frac{\left(\begin{array}{c}
N_{t}-1 \\
i
\end{array}\right)}{\left(N_{t}-i-1\right) !\left(N_{t}-i\right) !}\left(\frac{\bar{\gamma}_{1}\left(1-\left|\rho_{d}\right|^{2}\right)}{|\rho|^{2}}\right)^{i} \sum_{j=0}^{i N_{t}-i-1}\left(\begin{array}{c}
N_{t}-i-1 \\
j
\end{array}\right)\left(\frac{4}{\bar{\gamma}_{2}}\right)^{j} \\
& \times \frac{\Gamma\left(N_{t}-i+j+\frac{3}{2}\right) \Gamma\left(N_{t}-i-j-\frac{1}{2}\right)_{2} F_{1}\left(N_{t}-i+j+\frac{3}{2}, j+\frac{3}{2} ; N_{t}-i+1 ; \frac{\frac{1}{\bar{\gamma}_{1}}+\frac{1}{\frac{1}{\bar{\gamma}_{2}}+\frac{b}{2}-\frac{1}{\bar{\gamma}_{2}}+\frac{b}{2}+\frac{2}{\sqrt{\gamma_{1} \gamma_{2}}}}}{\sqrt{\bar{\gamma}_{1} \gamma_{2}}}\right)}{\left(\frac{1}{\bar{\gamma}_{1}}+\frac{1}{\bar{\gamma}_{2}}+\frac{b}{2}+\frac{2}{\sqrt{\bar{\gamma}_{1} \bar{\gamma}_{2}}}\right)^{N_{t}-i+j+\frac{3}{2}}} .
\end{aligned}
$$

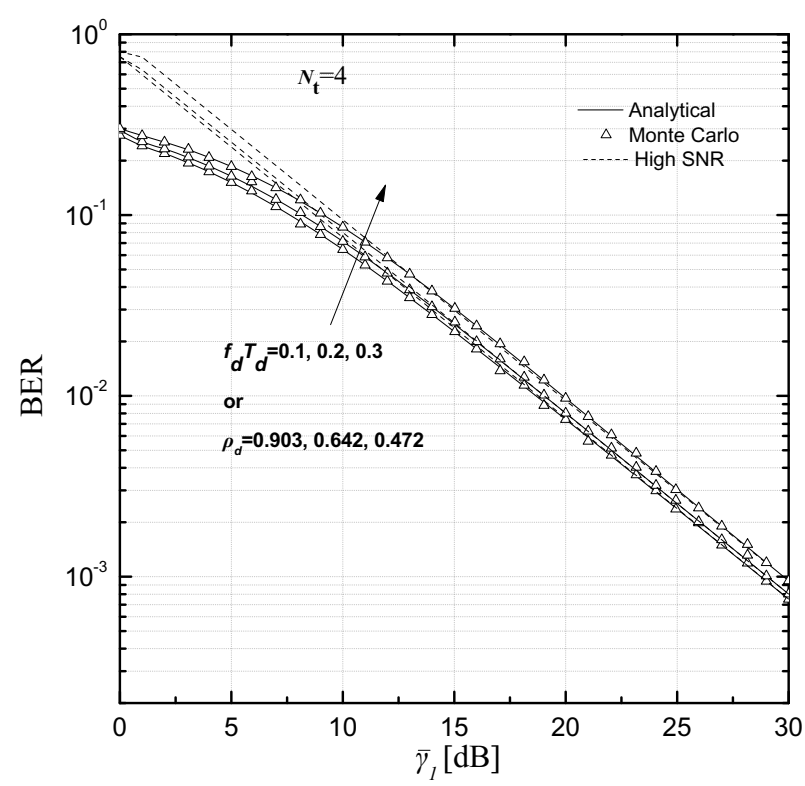

Fig. 4. Average BER using BPSK for $N_{t}=4, \bar{\gamma}_{1}=3 \bar{\gamma}_{2}$.

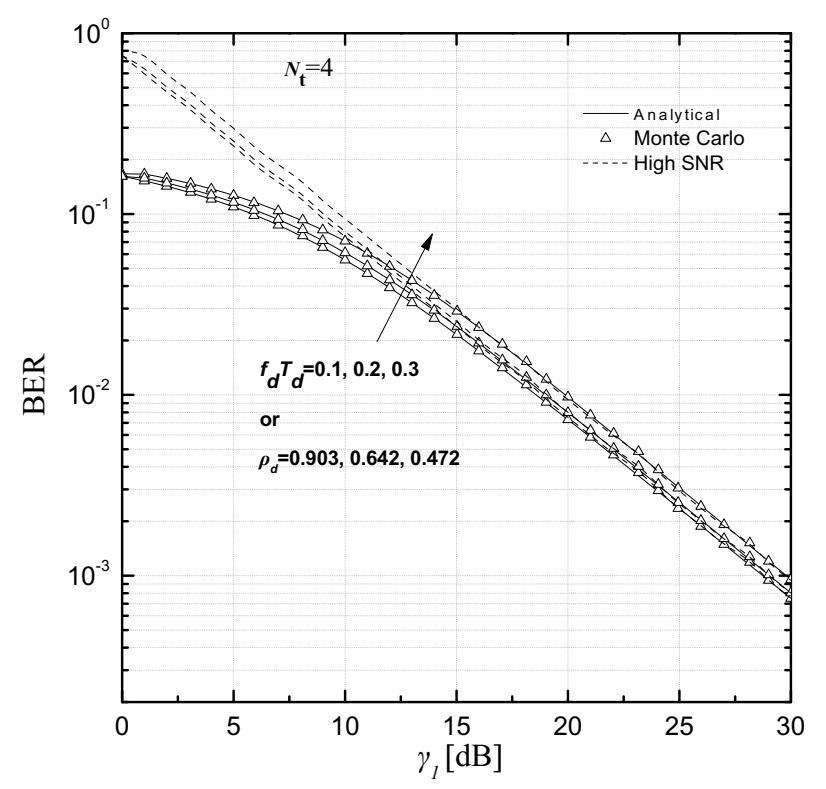

Fig. 5. Average BER using QPSK for $N_{t}=4, \bar{\gamma}_{1}=3 \bar{\gamma}_{2}$. behavior of the pdf of the output SNR vs. correlation coefficient $\rho_{d}$. We notice the agreement with the Monte Carlo simulations and that the pdf mean is a decreasing function of feedback delay $T_{d}$, i.e, the pdf curves shift to the high SNR region as the feedback delay decrease. This implies that the performance of the system is improved since it depends directly to the output SNR.

In Fig. 3 the outage probability of the output SNR is presented for various feedback delays. As for the pdf curves, the outage curves shift to high SNR values when the feedback delay tends to zero. Again the Monte Carlo simulation results confirm the accuracy of our mathematical analysis. As expected, the outage probability of the system degrades, as the system approaches an ideal situation where no feedback delay exists, i.e., $f_{d} T_{d}=0$.

In Figs. 4 and 5 system BER with BPSK and QPSK modulations is plotted, respectively. The analytical curves which clearly match the Monte Carlo simulations, are derived from (22), while the high-SNR curves from (24). Furthermore, we observe that the high-SNR approximation curves converge to the exact ones in the regimes of medium to high SNR and verifies that the diversity order of relaying beamforming systems with feedback delay is one. This result also confirms that feedback delays can cause significant system degradation compared to the perfect CSIT MIMO relaying with beamforming. Even if $N_{r}>1$ antennas are used at the destination, still the diversity order will be one compared to $\min \left(N_{t}, N_{r}\right)$ for a system with no feedback delays [10]. Hence, if the performance is not be sacrified much, more efficient feedback schemes to counteract the effect of feedback delay must be developed.

In practice, the beamforming performance of this $\mathrm{AF}$ relay system will further degrade due to finite rate feedback. Therefore, the presented analysis should be interpreted as a performance lower bound applicable in such scenarios. The chordal distance based formulation used in [5] to model finite rate feedback can be used to study the additional performance degradation. In some cases, finite rate quantization effects of the beamforming information vector could also be lumped into the flexible channel model adopted in this paper. That is, we could use an approximate correlation coefficient, $\tilde{\rho}$, to mimic the impact of quantization effects. A further extension of this current work could be to analyze the joint effects of feedback delay and quantization effects on the system performance.

\section{CONCLusions}

We have investigated the impact of outdated CSI due to feedback delay on the downlink performance of a two hop 
AF relay system with beamforming. In this system, the source is equipped with multiple antennas while the relay and the destination use a single antenna. We derived new closedform expressions for the statistics of the e2e SNR of this system. Next, exact average BER of the system for arbitrary SNR was derived. An accurate and simple BER approximation parameterized by the number of transmit antennas, $N_{t}$, and the correlation coefficient, $\rho_{d}$, is also derived to quantify the array gain at the high SNR region. Our analytical results are confirmed through comparison with Monte Carlo simulations.

\section{APPENDIX \\ Outage Probability at High SNR}

To obtain a first order expansion of $F_{\gamma_{e q 1}}\left(\gamma_{\text {th }}\right)$, we first note that

$$
\lim _{\bar{\gamma}_{1}, \bar{\gamma}_{2} \rightarrow \infty} F_{\gamma_{e q 1}}\left(\gamma_{\text {th }}\right)=\lim _{\bar{\gamma}_{1}, \bar{\gamma}_{2} \rightarrow \infty} F_{\gamma_{e q 2}}\left(\gamma_{\text {th }}\right)
$$

We substitute $\bar{\gamma}_{2}=\kappa \bar{\gamma}_{1}$ and $c=0$ into (15) and write the outage probability as

$$
\begin{aligned}
& F_{Y}\left(\gamma_{\mathrm{th}}\right)=1-\frac{2 e^{-\frac{(1+\kappa) \gamma_{\mathrm{th}}}{\kappa \gamma_{1}}} \gamma_{\mathrm{th}}^{N_{t}}}{\bar{\gamma}_{1}^{N_{t}}} \sum_{i=0}^{N_{t}-1}\left(\frac{\bar{\gamma}_{1}\left(1-\left|\rho_{d}\right|^{2}\right)}{\left|\rho_{d}\right|^{2} \gamma_{\mathrm{th}}}\right)^{i} \\
& \times \frac{\left(\left|\rho_{d}\right|^{2}\right)^{N_{t}-1}\left(\begin{array}{c}
N_{t}-1 \\
i
\end{array}\right)}{\left(N_{t}-i-1\right) !} \sum_{j=0}^{N_{t}-i-1} \frac{\left(\begin{array}{c}
N_{t}-i-1 \\
j
\end{array}\right)}{\kappa^{\frac{j+1}{2}}} \mathcal{K}_{j+1}\left(\frac{2 \gamma_{\mathrm{th}}}{\sqrt{\kappa} \bar{\gamma}_{1}}\right)
\end{aligned}
$$

Now by substituting $x=\frac{\gamma_{\text {th }}}{\bar{\gamma}_{1}}$ we can rewrite (29) as

$$
\begin{aligned}
& F_{Y}(x)=1-2 e^{-\frac{1+\kappa}{\kappa}} x \sum_{i=0}^{N_{t}-1} \frac{\left(\begin{array}{c}
N_{t}-1 \\
i
\end{array}\right)\left(\frac{1-\left|\rho_{d}\right|^{2}}{\left|\rho_{d}\right|^{2}}\right)^{i}}{\left(N_{t}-i-1\right) !} \\
& \times\left(\left|\rho_{d}\right|^{2}\right)^{N_{t}-1} \sum_{j=0}^{N_{t}-i-1} \frac{\left(\begin{array}{c}
N_{t}-i-1 \\
j
\end{array}\right)}{\kappa^{\frac{j+1}{2}}} x^{N_{t}-i} \mathcal{K}_{j+1}\left(\frac{2 x}{\sqrt{\kappa}}\right)
\end{aligned}
$$

When $\nu>0$ is fixed and $x \rightarrow 0$ the following approximation holds from [15, Eq. (9.6.9)]

$$
\mathcal{K}_{\nu}(x) \simeq \frac{2^{\nu-1} \Gamma(\nu)}{x^{\nu}}
$$

Therefore, applying (31) in (30) we get

$$
\begin{aligned}
F_{Y}(x) & =1-e^{-\frac{1+\kappa}{\kappa} x} \sum_{i=0}^{N_{t}-1}\left(\begin{array}{c}
N_{t}-1 \\
i
\end{array}\right)\left(\frac{1-\left|\rho_{d}\right|^{2}}{\left|\rho_{d}\right|^{2}}\right)^{i} \\
& \times\left(\left|\rho_{d}\right|^{2}\right)^{N_{t}-1} \sum_{j=0}^{N_{t}-i-1} \frac{x^{N_{t}-i-j-1}}{\left(N_{t}-i-j-1\right) !}
\end{aligned}
$$

Using the McLaurin series representation for the exponential function in (32) yields

$$
\begin{aligned}
& F_{Y}(x)=1-\left(\left|\rho_{d}\right|^{2}\right)^{N_{t}-1} \sum_{p=0}^{\infty} \frac{\left(-\frac{1+\kappa}{\kappa} x\right)^{p}}{p !} \\
& \times \sum_{i=0}^{N_{t}-1}\left(\begin{array}{c}
N_{t}-1 \\
i
\end{array}\right)\left(\frac{1-\left|\rho_{d}\right|^{2}}{\left|\rho_{d}\right|^{2}}\right)^{i} \sum_{j=0}^{N_{t}-i-1} \frac{x^{N_{t}-i-j-1}}{\left(N_{t}-i-j-1\right) !}
\end{aligned}
$$

Collecting only the first order terms yields

$$
\begin{aligned}
F_{Y}(x) & =1-\left(\left|\rho_{d}\right|^{2}\right)^{N_{t}-1}\left(1-\frac{1+\kappa}{\kappa} x+O(x)\right) \\
& \times\left(\sum_{i=0}^{N_{t}-1}\left(\begin{array}{c}
N_{t}-1 \\
i
\end{array}\right)\left(\frac{1-\left|\rho_{d}\right|^{2}}{\left|\rho_{d}\right|^{2}}\right)^{i}\right. \\
& \left.+\sum_{i=0}^{N_{t}-2}\left(\begin{array}{c}
N_{t}-1 \\
i
\end{array}\right)\left(\frac{1-\left|\rho_{d}\right|^{2}}{\left|\rho_{d}\right|^{2}}\right)^{i} x+O(x)\right)
\end{aligned}
$$

Simplifying (34) we get

$$
F_{Y}(x)=\left(\frac{1+\kappa}{\kappa}-\left(1-\left(1-\left|\rho_{d}\right|^{2}\right)^{N_{t}-1}\right)\right) x
$$

We see that (35) accurately describes the behavior of $F_{Y}(x)$ around $x=0$. By substituting $x=\frac{\gamma_{\text {th }}}{\bar{\gamma}_{1}}$ into (35), the desired result can be obtained.

\section{REFERENCES}

[1] J. Boyer, D. D. Falconer and H. Yanikomeroglu, "Multihop diversity in wireless relaying channels," IEEE Trans. Commun., vol. 52, pp. 18201830, Oct. 2004.

[2] M. O. Hasna and M.-S. Alouini, "A performance study of dual-hop transmissions with fixed gain relays," IEEE Trans. Wireless Commun., vol. 3, pp. 1963-1968, Nov. 2004.

[3] H. Mheidat and M. Uysal, "Impact of receive diversity on the performance of amplify-and-forward relaying under APS and IPS power constraints," IEEE Commun. Lett., vol. 10, pp. 468-470, June 2006.

[4] D. J. Love, R. W. Heath, Jr., V. K. N. Lau, D. Gesbert, B. D. Rao, and M. Andrews, "Overview of limited feedback in wireless communication systems," IEEE J. Selected Areas Commun., vol. 26, pp. 1341-1365, Oct. 2008.

[5] Y. Ma and D. Zhang, A Leith and Z. Wang "Error performance of transmit beamforming with delayed and limited feedback," IEEE Trans. Wireless Commun., vol. 8, pp. 1164-1170, March 2009.

[6] Y. Fan and J. Thompson, "MIMO configurations for relay channels: Theory and practice," IEEE Trans. Wireless Commun., vol. 6, pp. 17741786, May 2007.

[7] Y. Zhao, R. Adve and T. J. Lim, "Beamforming with limited feedback in amplify-and-forward cooperative networks," IEEE Trans. Wireless Commun., vol. 7, pp. 5145-5149, Dec. 2008.

[8] Z. Ding, W. H. Chin and K. K. Leung, "Distributed beamforming and power allocation for cooperative networks," IEEE Trans. Wireless Commun., vol. 7, pp. 1817-1822, May 2008.

[9] Y. Fan, A. Adinoyi, J. S. Thompson, H. Yanikomeroglu and H. V. Poor, "A simple distributed antenna combining approach for cooperative

[10] R. H. Y. Louie, Y. Li, and B. Vucetic, "Performance analysis of beamforming in two hop amplify and forward relay networks," in Proc. IEEE ICC 2008, Beijing, China, May 2008, pp. 4311-4315.

[11] C. Lee, J. Joung and Y. H. Lee, "A pilot emitting amplify-and-forward relay and its application to hop-by-hop beamforming," in Proc. IEEE PIMRC 2008, Cannes, France, Sept. 2008.

[12] E. Zeng, S. Zhu, X. Liao, Z. Zhong and Z. Feng, "On the performance of amplify-and-forward relay systems with limited feedback beamforming," IEICE Trans. Commun., vol. E91-B, pp. 2053-2057, June 2008.

[13] H. Min, S. Lee, K. Kwak and D. Hong, "Effect of multiple antennas at the source on outage probability for amplify-and-forward relaying systems," IEEE Trans. Wireless Commun., vol. 8, pp. 633-637, Feb. 2009.

[14] I. S. Gradshteyn and I. M. Ryzhik, Table of Integrals, Series and Products. 6th ed., San Diego: CA, Academic Press, 2000.

[15] M. Abramovitz and I. A. Stegun, Handbook of Mathematical Functions with Formulas, Graphs, and Mathematical Tables, 9th ed. New York: Dover, 1972.

[16] Z. Wang and G. B. Giannakis, "A simple and general parameterization quantifying performance in fading channels," IEEE Trans. Commun., vol. 51, pp. 1389-1398, Aug. 2003. diversity," IEEE Trans. Commun., vol. 57, pp. 626-629, March 2009. 